\title{
Metagenomic Analysis of Serum Microbe-Derived Extracellular Vesicles and Diagnostic Models to Differentiate Ovarian Cancer and Benign Ovarian Tumor
}

\author{
Se Ik Kim $\left.{ }^{1,+} \mathbb{(}\right)$, Nayeon Kang ${ }^{2,+}$, Sangseob Leem ${ }^{3}$, Jinho Yang ${ }^{4}{ }^{(}$, HyunA Jo ${ }^{5}$, Maria Lee ${ }^{1}{ }^{(D}$, \\ Hee Seung Kim ${ }^{1}{ }^{\mathbb{D}}$, Danny N. Dhanasekaran ${ }^{6}$, Yoon-Keun Kim ${ }^{4}$, Taesung Park ${ }^{2, *}$ and \\ Yong Sang Song $1,5, * \mathbb{D}$ \\ 1 Department of Obstetrics and Gynecology, Seoul National University College of Medicine, Seoul 03080, \\ Korea; seikky@naver.com (S.I.K.); marialeemd@gmail.com (M.L.); bboddi0311@gmail.com (H.S.K.) \\ 2 Department of Statistics, Seoul National University, Seoul 08826, Korea; thisisnykang@gmail.com \\ 3 Department of Core Technology, R\&D Center, LG Household \& Healthcare, Seoul 07795, Korea; \\ leemss@ajou.ac.kr \\ 4 MD Healthcare Inc., Seoul 03923, Korea; jhyang@mdhc.kr (J.Y.); ykkim@mdhc.kr (Y.-K.K.) \\ 5 Cancer Research Institute, Seoul National University College of Medicine, Seoul 03080, Korea; \\ whgusdk25@snu.ac.kr \\ 6 Stephenson Cancer Center, University of Oklahoma Health Sciences Center, Oklahoma City, OK 73104, USA; \\ Danny-Dhanasekaran@ouhsc.edu \\ * Correspondence: tspark@stats.snu.ac.kr (T.P.); yssong@snu.ac.kr (Y.S.S.); \\ Tel.: +82-2-880-8924 (T.P.); +82-2-2072-2822 (Y.S.S.) \\ + These two authors contributed equally as first authors.
}

Received: 7 April 2020; Accepted: 19 May 2020; Published: 21 May 2020

check for updates

\begin{abstract}
We aimed to develop a diagnostic model identifying ovarian cancer (OC) from benign ovarian tumors using metagenomic data from serum microbe-derived extracellular vesicles (EVs). We obtained serum samples from 166 patients with pathologically confirmed OC and 76 patients with benign ovarian tumors. For model construction and validation, samples were randomly divided into training and test sets in the ratio 2:1. Isolation of microbial EVs from serum samples of the patients and $16 \mathrm{~S}$ rDNA amplicon sequencing were carried out. Metagenomic and clinicopathologic data-based OC diagnostic models were constructed in the training set and then validated in the test set. There were significant differences in the metagenomic profiles between the OC and benign ovarian tumor groups; specifically, genus Acinetobacter was significantly more abundant in the OC group. More importantly, Acinetobacter was the only common genus identified by seven different statistical analysis methods. Among the various metagenomic and clinicopathologic data-based OC diagnostic models, the model consisting of age, serum CA-125 levels, and relative abundance of Acinetobacter showed the best diagnostic performance with the area under the receiver operating characteristic curve of 0.898 and 0.846 in the training and test sets, respectively. Thus, our findings establish a metagenomic analysis of serum microbe-derived EVs as a potential tool for the diagnosis of OC.
\end{abstract}

Keywords: ovarian neoplasms; ovarian carcinoma; extracellular vesicle; microbiome; metagenomic analysis; diagnostic model 


\section{Introduction}

Ovarian cancer (OC) is the deadliest gynecologic cancer worldwide [1]. In the United States, the number of new cases of OC and cancer deaths from OC in 2019 were estimated to be $22,530(2.5 \%$ of all female cancers) and 13,980 (4.9\% of female cancer deaths), respectively [2]. In Korea, the incidence of OC has been increasing gradually [3]. Owing to a lack of specific symptoms and effective screening tools, the majority of $\mathrm{OC}$ cases are diagnosed at an advanced stage, resulting in a high recurrence and mortality rate [4]. Among the various histologic types of OC, the majority (90\%) are epithelial OC.

For women with adnexal masses, distinguishing OC from benign ovarian tumors is an important issue, as it determines the treatment plan, including the surgical approach. The detection tools currently available for OC are serum cancer antigen 125 (CA-125) levels, ultrasonography, computed tomography (CT) scans, and magnetic resonance imaging (MRI). Combinations of modalities provide better diagnostic performance for identifying OC than each modality alone [5]. The risk of malignancy index (RMI) scoring system, consisting of serum CA-125, menopausal status, and ultrasound features, as well as the risk of ovarian malignancy algorithm (ROMA), a biomarker-based algorithm consisting of serum CA-125 and human epididymis protein 4 (HE4), have been developed [6,7]. Both RMI and ROMA are reliable tools and perform equally well in differentiating OC from adnexal masses $[8,9]$. However, considering that their diagnostic performance and accuracy differ among the prospective cohort studies, further improvements in differentiating adnexal masses are still needed [10-12].

Microbiota, a microbial environmental factor that we are constantly exposed to, has emerged as a link between the host and various cancer types. Human microbiome studies have revealed that significant differences in microbiota composition are associated with oral, esophageal, pancreatic, and colorectal cancers [13-16]. Although the exact underlying mechanisms are still not well-understood, microbe-induced inflammation is thought to trigger changes in the tumor microenvironment, promoting tumorigenesis $[17,18]$. Advances in the sequencing technique of microbial genomes have expanded microbiome data and extended our understanding on microbiota-host interactions. Especially, 20-200 nanometer-sized extracellular vesicles (EVs), constitutively secreted by microbes and detectable in body fluids, are considered to play an important role in such interactions $[19,20]$.

A recent study has compared the microbiome signature between fresh OC tissues $(n=25)$ and normal fallopian tube fimbria tissues $(n=25)$ and suggested that changes in microbial composition might be related to the process of OC development [21]. However, the relationship between serum microbial EVs and OC has yet to be investigated. The relative abundance of certain microbial EVs released in the blood might differ between benign and malignant ovarian tumors, and those differences could be utilized in the differential diagnosis of adnexal masses. Thus, this study aimed at developing diagnostic models to differentiate between $\mathrm{OC}$ and benign ovarian tumors through the metagenomic analysis of serum microbial EVs.

\section{Results}

\subsection{Characteristics of the Study Population}

The overall study design is displayed in Figure S1. Table 1 presents the clinicopathologic characteristics of all patients. Although patients in the OC group were significantly older than those in the benign ovarian tumor group (mean, 53.6 vs. 49.4 years; $p=0.041$ ), other characteristics such as body mass index (BMI), menopausal status, and comorbidities were similar. After 2:1 random distribution of the patients into training and test sets, OC patients were still older than those with benign ovarian tumors in the training set, whereas patients' ages were similar in the test set. Both in the training and test sets, no differences in BMI, menopausal status, and comorbidities were observed between the OC and benign ovarian tumor groups. 
Table 1. Patients' clinicopathologic characteristics.

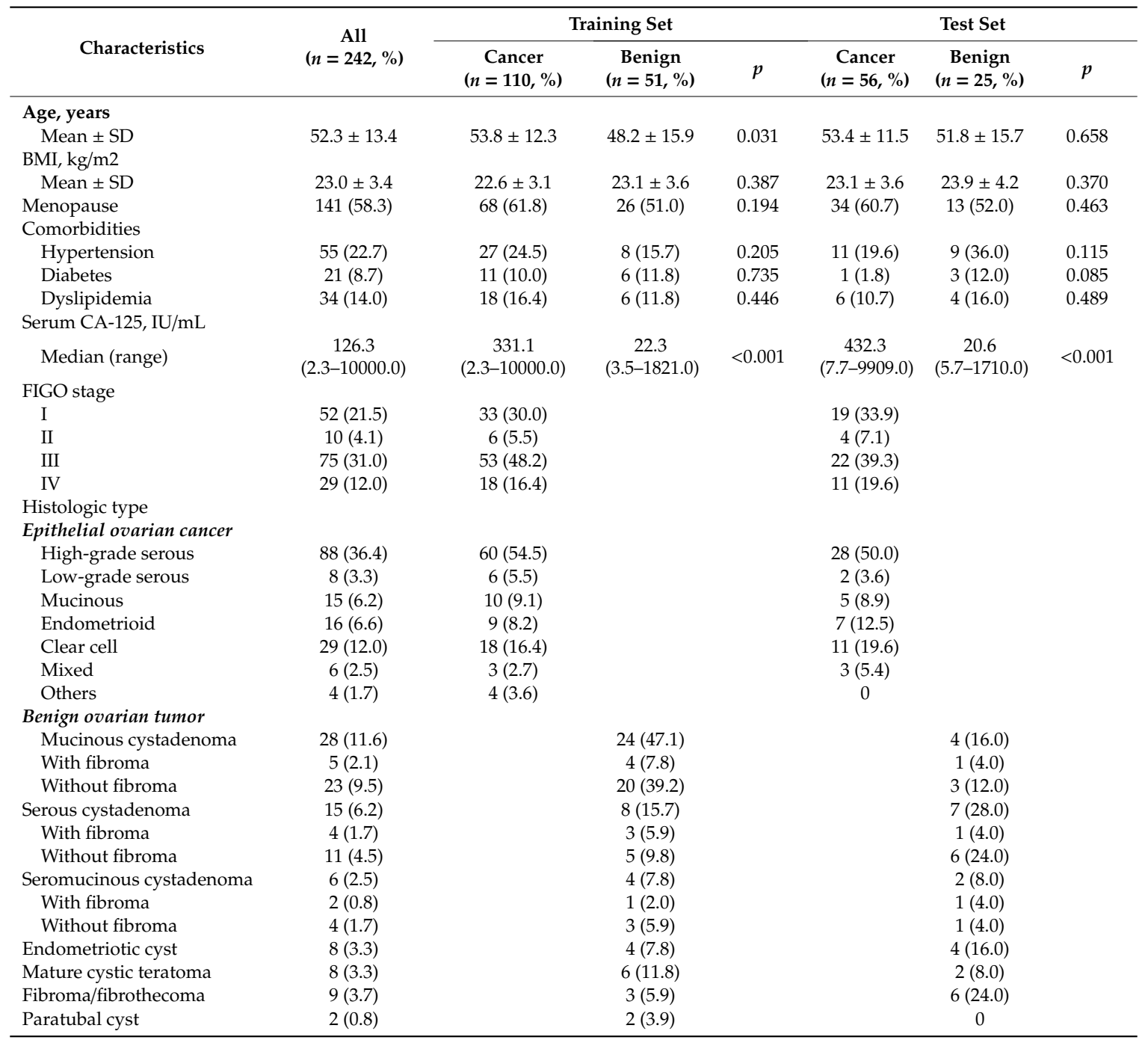

Abbreviations: BMI, body mass index; CA-125, cancer antigen 125; FIGO, International Federation of Gynecology and Obstetrics; and SD, standard deviation.

In the training set, serum CA-125 levels were significantly higher in patients with OC (median, 331.1 vs. $22.3 \mathrm{IU} / \mathrm{mL} ; p<0.001)$. Among the 110 patients with OC, $39(35.5 \%)$ and $71(64.5 \%)$ were diagnosed with the International Federation of Gynecology and Obstetrics (FIGO) stage I-II and III-IV, respectively. The most common histologic type was high-grade serous carcinoma, which was observed in $54.5 \%$ of OC patients. Among the 51 patients with benign ovarian tumor, mucinous cystadenoma (47.1\%) was the most common pathologic diagnosis, followed by serous cystadenoma (15.7\%).

In the test set, the OC group also showed significantly higher serum CA-125 levels compared to the benign ovarian tumor group (median, 432.3 vs. $20.6 \mathrm{IU} / \mathrm{mL} ; p<0.001$ ). FIGO stage I-II disease was observed in $41.1 \%$ of OC patients. The most common histologic types in the OC and benign ovarian tumor groups were high-grade serous carcinoma (50.0\%) and serous cystadenoma (28.0\%), respectively.

\subsection{Comparison of Metagenomic Profiles between the Two Groups}

Figure 1 depicts the landscape of the metagenomic profiles of all patients. Figure 1A shows all 31 phyla detected in OC and benign ovarian tumor groups. In the genus-level composition, a total of 587 genera were detected in all patients. Among them, 110 significantly differentially distributed genera identified by at least two statistical methods are displayed with their relative abundance in Figure $1 \mathrm{~B}$. Herein, genus Acinetobacter showed high relative abundances both in $\mathrm{OC}$ and benign ovarian tumor 
groups. In the training set, 107 of 110 ovarian cancer patients (97.3\%) had Acinetobacter, while 50 of 51 benign ovarian tumor patients $(98.0 \%)$ had Acinetobacter. In the test set, Acinetobacter was found in $98.2 \%$ (55/56) and $100.0 \%$ (25/25) of OC and benign ovarian tumor groups, respectively.

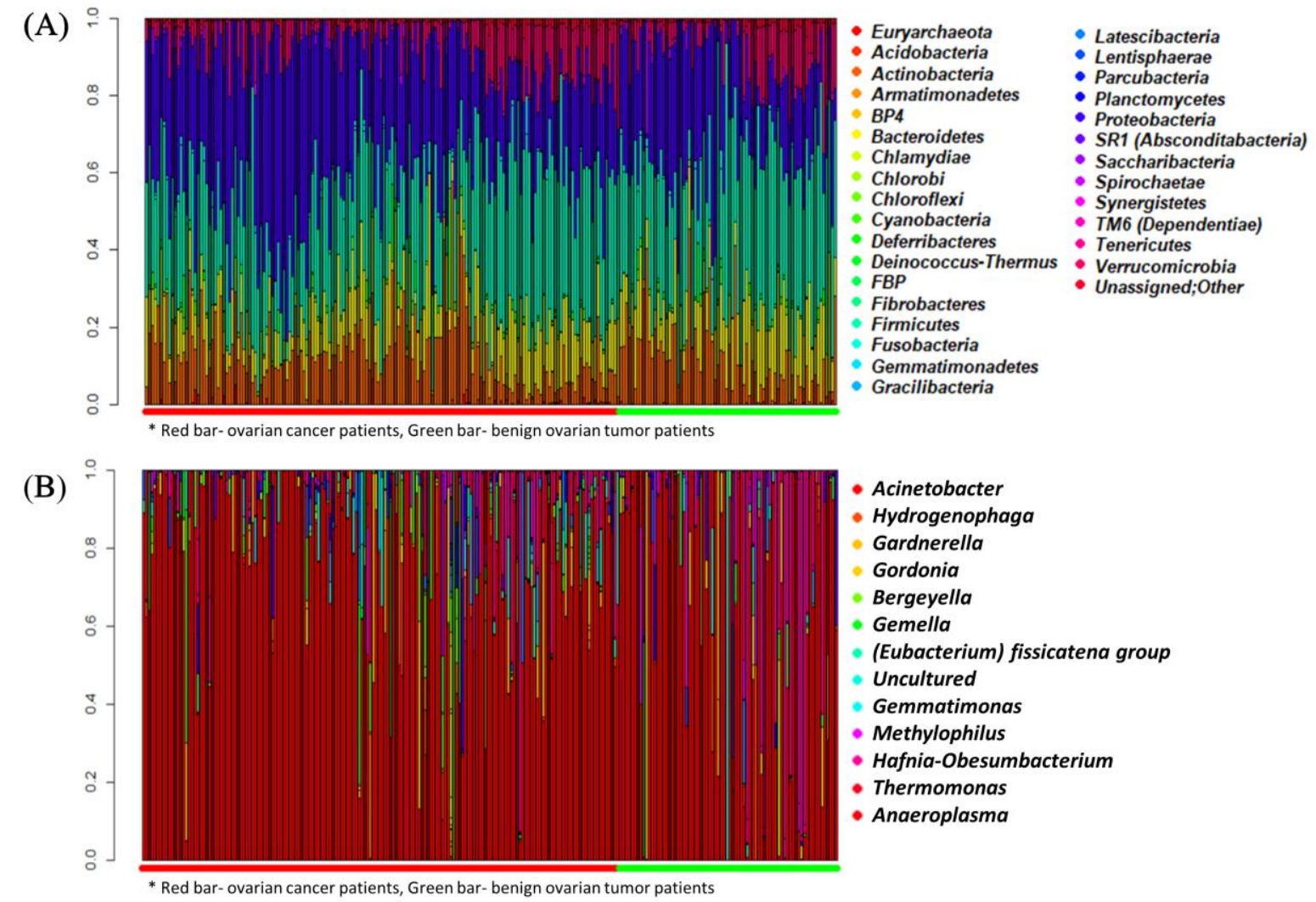

Figure 1. Landscape of metagenomic profiles in all patients. (A) Phylum-level composition. (B) Genus-level composition. Below the plot, red and green horizontal bars indicate ovarian cancer patients and benign ovarian tumor patients, respectively.

In general, "genus" is regarded as the lowest level of taxonomy, where unassigned or unclassified microbiome are relatively small. Most previous studies on microbiome analyses have reported metagenomic profiles up to the genus level. Therefore, we investigated further metagenomic profiles of the two groups in the genus level.

In metagenomics, $\alpha$-diversity and $\beta$-diversity are used to overview the distribution of the data composition: $\alpha$-diversity refers to the richness, evenness, and dominance of taxa in a particular community, while $\beta$-diversity means taxonomic differences between the communities [22]. Comparing the genus-level $\alpha$-diversity, the Shannon index was not different between the OC and benign ovarian tumor groups in the training set (median, 3.294 vs. 3.263; $p=0.270$ ), as well as in the test set (median, 3.210 vs. $3.238 ; p=0.810$ ) (Figure S2). In order to compare $\beta$-diversity, we analyzed clustering at the genus level using multidimensional plots. However, these plots did not show distinguished clustering between the OC and benign ovarian tumor groups in the training and test sets (Figure S3).

\subsection{Development of Diagnostic Models for Ovarian Cancer in the Training Set}

Through the metagenomic analyses using various statistical methods, we identified genus-level microbiome biomarkers that were differentially distributed between the OC and benign ovarian tumor groups with statistical significance: Wilcoxon test, Metastats, EdgeR, DESeq2, zero-inflated Gaussian mixture model (ZIG), zero-inflated beta regression (ZIBSeq), analysis of composition of microbiomes (ANCOM), and centered log-ratio transformation and permutation logistic regression model (CLR 
Perm) identified 1, 98, 3, 8, 447, 56, 1, and 2 biomarkers, respectively, at adjusted $q$ values using a false discovery rate $(\leq 0.05)$. Table 2 shows the top 10 microbiome biomarkers identified by each statistical method, in order of overlap.

Table 2. Top 10 genus-level microbiome biomarkers significantly differentially distributed between the ovarian cancer and benign ovarian tumor groups.

\begin{tabular}{ccccccccc}
\hline Genus & Wilcoxon & Metastats & EdgeR & DESeq2 & ZIG & ZIBSeq & ANCOM & CLR Perm \\
\hline Acinetobacter & $<0.001$ & 0.008 & 0.093 & 0.043 & $<0.001$ & $<0.001$ & Acinetobacter & $<0.001$ \\
Isoptericola & 0.841 & $<0.001$ & 0.487 & 1 & 0.046 & $<0.001$ & Not detected & 0.855 \\
Terrisporobacter & 0.841 & 0.023 & 0.600 & 1 & $<0.001$ & $<0.001$ & Not detected & 0.944 \\
SM1A02 & 0.989 & 0.008 & 0.528 & 1 & $<0.001$ & 0.002 & Not detected & 0.935 \\
Candidatus Alysiosphaera & 0.841 & $<0.001$ & 0.476 & 1 & 0.015 & $<0.001$ & Not detected & 0.901 \\
Ralstonia & 0.841 & $<0.001$ & 0.462 & 1 & $<0.001$ & 0.005 & Not detected & 0.913 \\
Hydrogenophaga & 0.771 & $<0.001$ & 0.872 & 1 & $<0.001$ & 0.027 & Not detected & 0.809 \\
Pseudorhodoferax & 0.921 & 0.024 & 0.811 & 1 & $<0.001$ & 0.007 & Not detected & 0.779 \\
Bryobacter & 0.841 & 0.023 & 0.420 & 1 & 0.007 & 0.999 & Not detected & 0.849 \\
Varibaculum & 0.841 & 0.013 & 0.600 & 1 & 0.599 & $<0.001$ & Not detected & 0.416 \\
\hline
\end{tabular}

Shown with the $q$ values.

Next, we examined the overlap of these genus-level microbiome biomarkers among the eight statistical methods (Figure 2). In total, 486 biomarkers were identified to be significantly differentially distributed by at least one statistical method. Among them, 110 and nine markers overlapped at least two and three statistical methods, respectively. Acinetobacter was the only common genus identified by seven different statistical analysis methods. Specifically, Acinetobacter was significantly more abundant in the OC group than in the benign ovarian tumor group (median (interquartile range), $0.084(0.037-0.222)$ vs. $0.033(0.008-0.075)$; Wilcoxon rank sum test, $p<0.001)$. Therefore, we selected Acinetobacter as the most potential and highly plausible genus-level microbiome biomarker for differentiating OC from benign ovarian tumors.

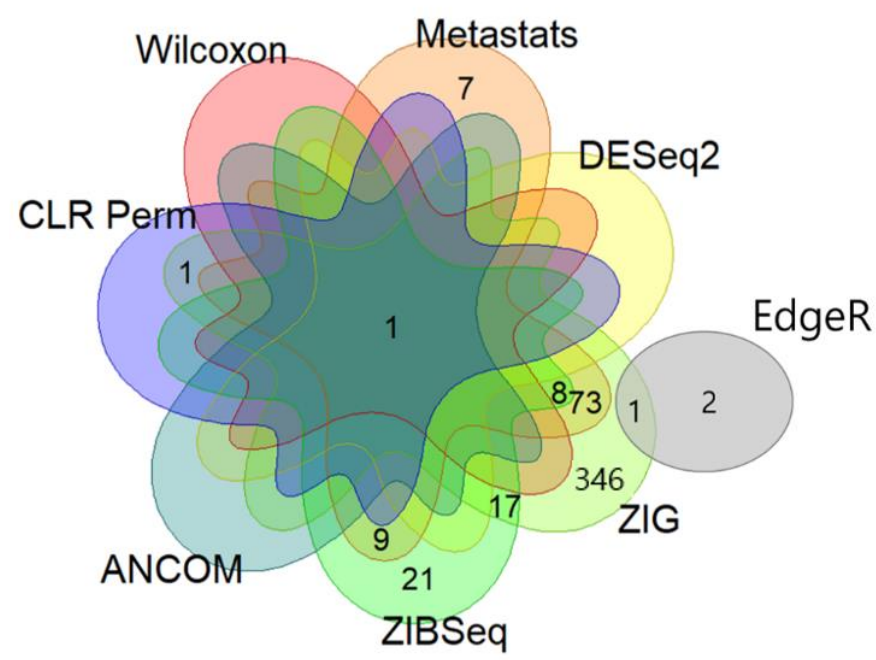

Figure 2. Selection of genus-level microbiome biomarkers. Venn diagram depicts the overlapping of biomarkers among the eight statistical methods.

Combining the relative abundance of Acinetobacter with patients' clinicopathologic variables, we constructed several diagnostic models to differentiate $\mathrm{OC}$ from benign ovarian tumors (Table 3). The model composed of age, serum CA-125 levels, and relative abundance of Acinetobacter showed $86.4 \%$ sensitivity and $78.4 \%$ specificity. This model showed a superior area under the receiver operating characteristic curve (AUC; 0.898 ) than any other models, with less than three of the following variables: age, serum CA-125 levels, and Acinetobacter. 
Table 3. Diagnostic models differentiating ovarian cancer from benign ovarian tumors.

\begin{tabular}{ccccccc}
\hline \multirow{2}{*}{ Model } & \multicolumn{3}{c}{ Training Set } & \multicolumn{3}{c}{ Test Set } \\
\cline { 2 - 7 } & Sensitivity & Specificity & AUC & Sensitivity & Specificity & AUC \\
\hline Age & 0.554 & 0.490 & 0.589 & 0.518 & 0.520 & 0.531 \\
Age, CA-125 & 0.773 & 0.686 & 0.809 & 0.768 & 0.560 & 0.816 \\
Age, Acinetobacter & 0.827 & 0.529 & 0.770 & 0.839 & 0.440 & 0.667 \\
Age, CA-125, & 0.864 & 0.784 & 0.898 & 0.821 & 0.680 & 0.846 \\
Acinetobacter & & & & & & \\
\hline
\end{tabular}

Abbreviations: AUC, area under the receiver operating characteristic curve (AUC) and CA-125, cancer antigen 125.

\subsection{Validation of Diagnostic Models for Ovarian Cancer}

The developed diagnostic models were validated in the test set. Among the various models, the model consisting of patients' ages at diagnosis, initial serum CA-125 levels, and relative abundance of Acinetobacter yielded the best diagnostic performance for differentiating OC from benign ovarian tumors as follows: sensitivity, $82.1 \%$; specificity, $68.0 \%$; and AUC, 0.846 (Table 3 and Figure 3).

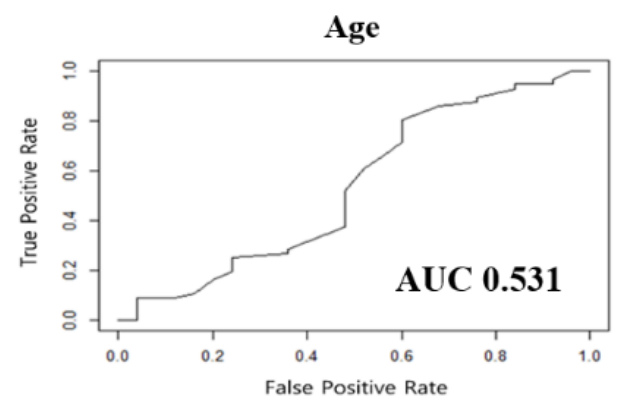

Age, Acinetobacter

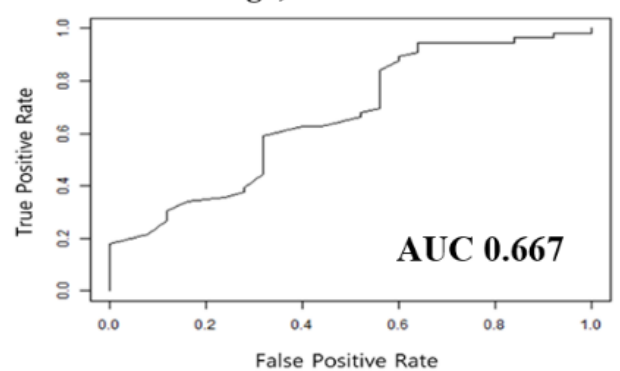

Age, CA-125

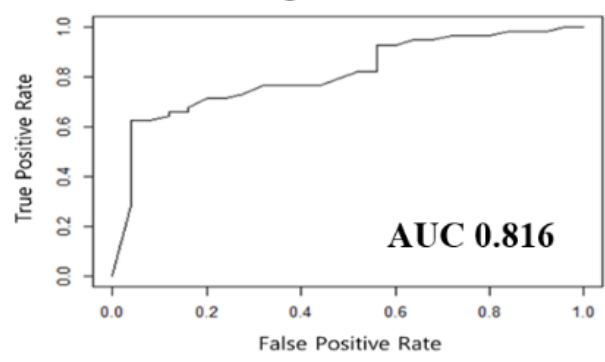

Age, CA-125, Acinetobacter

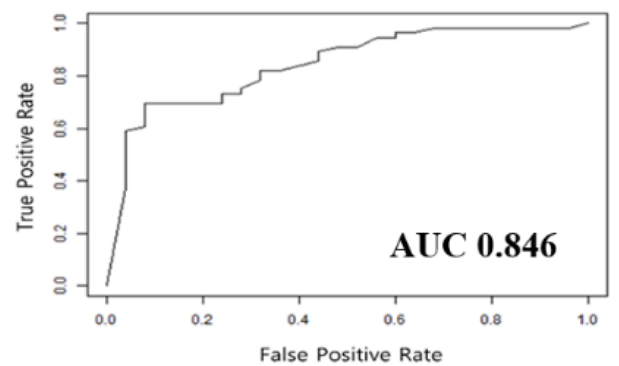

Figure 3. Comparisons of performances among diagnostic models differentiating ovarian cancer from benign ovarian tumors.

\section{Discussion}

In the present study, we successfully extracted microbe-derived EVs from the serum samples and characterized the metagenomic profiles of 242 patients: 166 with OC and 76 with benign ovarian tumors. Incorporating the relative abundance of specific microbiomes at the genus level with patients' ages and serum CA-125 levels, we developed a new diagnostic model to differentiate OC from benign ovarian tumors; this model even showed a better diagnostic performance than those without a microbiome biomarker.

Recently, metagenomic analysis has been noticed as a new approach; it has opened new horizons in the diagnosis of human disease. The Human Microbiome Project, funded by the National Institutes of Health, triggered the broadening of our insights into the microbiome. The relative abundance of certain microbes varies in chronic diseases, such as diabetes, obesity, cardiovascular disease, inflammatory bowel disease, and chronic allergies $[23,24]$. In various malignancies, disruption in the stability of 
microbiota or structural microbiome shifts have been reported [13-16]. However, to date, few studies have examined microbiomes in OC [21].

The current study provides new scientific evidence regarding different distributions of microbiomes in serum EVs between OC and benign ovarian tumors. Unlike previous researchers who used samples obtained from female reproductive organs [21,25], we used patients' serum samples. Compared to the former, obtaining serum samples is much easier and less invasive; organ harvesting is not required. Considering the fact that an exact diagnosis is confirmed through surgery, a preoperative diagnostic model using serum samples certainly has merit. Therefore, our study shows the potential of serum microbial EVs as a liquid biopsy for the diagnosis of OC.

Interestingly, we found that the genus Acinetobacter was significantly more abundant in the OC group than in the benign ovarian tumor group. Moreover, Acinetobacter was the only commonly found genus through almost all available statistical analysis methods developed so far. In general, Acinetobacter baumannii (A. baumannii), a species of the genus Acinetobacter, is a pathogen related to human infections, such as pneumonia, blood stream infection, urinary tract infection, and meningitis [26]. Infection with Acinetobacter is also common in cancer patients, and a relationship between A. baumannii and poor survival outcomes was also reported among patients with various cancer types [27,28]. Similar to our study, Zhou et al. showed that Acinetobacter, especially the Acinetobacter lwoffii species, was significantly enriched in OC tissues compared to normal distal fallopian tube tissues [21].

To explore the underlying mechanisms between Acinetobacter and epithelial OC, the following two aspects should be considered: bacterial factors and host responses against them. Bacterial products, such as lipopolysaccharide (LPS), can stimulate the tumor to produce proinflammatory cytokines that enhance tumor survival (LPS-induced tumor growth). On the host side, Toll-like receptors (TLRs) are transmembrane proteins known to play an important role in immunosurveillance and responses toward microorganisms [29].

Previously, through in vitro and in vivo studies, researchers have demonstrated that LPS, as well as EVs, secreted by A. baumannii stimulate the TLR-4 signaling pathway and trigger the host's immune response against an A. baumannii infection [30-33]. In addition, EVs secreted by Acinetobacter nosocomialis, another important pathogen of various opportunistic infections, are also known to induce cytotoxicity of epithelial cells and host inflammatory responses [34]. Interestingly, the expression of TLR-4 is observed in both the normal ovarian surface and epithelial ovarian tumor cell lines [35]. In epithelial OC, TLR-4 signaling has been demonstrated to promote tumor growth and to develop chemoresistance [36]. Therefore, we suggest that products secreted by the Acinetobacter species may cause the development of epithelial OC through the TLR-4 signaling pathway.

In accordance with the era of precision medicine, it is obvious that reliable diagnostic tools are essential for detecting OC. Our study results imply that adding the metagenomic data to the conventional diagnostic model might improve its performance in the detection of OC. However, the diagnostic model composed of patients' ages, serum CA-125 levels, and relative abundance of the genus Acinetobacter needs to be externally validated. Nevertheless, this study tried to overcome this limitation by separating the test and training sets from the beginning and faithfully implementing the internal validation.

Developing the diagnostic models for identifying OC, we believed that it was the most important to reduce the false-negative rate considering its worse prognosis compared to any other malignancies. Therefore, during the model construction, we focused on achieving a high accuracy and maintaining the sensitivity, even if specificity was compromised. As the result, we reported our newly developed diagnostic model's diagnostic performance as follows: sensitivity $86.4 \%$ and specificity $78.4 \%$ (AUC 0.898 ) in the training set; and sensitivity $82.1 \%$ and specificity $68.0 \%$ (AUC 0.846 ) in the test set.

Diagnostic performance of our newly developed, microbiome biomarker-based diagnostic model was not compared with the currently available tools, such as the ROMA and RMI scoring systems. At our institution, the serum HE4 test is not routinely performed in women with adnexal masses. In our study population, only 53.3\% (129/242) underwent the serum HE4 test, so that ROMA could be 
calculated. Owing to the retrospective study design, we were not able to retrieve all the preoperative transvaginal ultrasonography images, so that RMI scoring system could not be applied. Moreover, if microbiome biomarkers are integrated with ROMA or RMI, there is the possibility that the diagnostic performance for identifying OC might be much improved. Now, we are planning a prospective cohort study to validate the clinical usefulness of the serum-based metagenomic analysis in the diagnosis of OC. In that study, every single subject will undergo both ROMA and RMI for further investigation.

The current study also has other limitations. First, the relationship between the microbiome and OC should be further investigated. We do not know whether our findings could explain the pathogenesis of OC or were just a phenomenon in this cohort. The cause-and-effect relationship between differing microbiome compositions and $\mathrm{OC}$ should be investigated. Additional translational studies, such as hypothesis-proving cell-line or animal studies, are warranted. Second, the current study is a single-institution study requiring external validation in different study populations. For example, the proportion of clear cell carcinoma in the OC group was relatively high: $16.4 \%$ and $19.6 \%$ in the training and test sets, respectively. In this study, all patients were Korean, and according to the literature on histologic types of epithelial $O C$, ovarian clear cell carcinoma is more common in the East Asian population than in the Western population [37,38]. Therefore, OC groups from other regions or ethnicities with different proportions of histologic types might have different metagenomic profiles of serum EVs. Third, the the FIGO stage of the OC cases was not considered in developing the diagnostic models. Approximately $30 \%$ of OC patients in our study population had FIGO stage I disease. The extent of disease might affect the composition of the serum microbe-derived EVs. Therefore, it is necessary to compare OC patients' metagenomic profiles by stages in a large-sized cohort. Lastly, the sample size for the benign ovarian tumor group was small, which resulted in quite different histologic types between the training and test sets, although we randomly divided the samples.

Despite these limitations, the current study was the first to characterize the metagenomic profiles of the serum microbial EVs in OC. Through evaluation of the serum microbiomes, we were able to build a diagnostic model for OC. The metagenomic analysis of serum microbiomes has several advantages, particularly the ease of sample collection, which suggests an increase of its usability.

\section{Materials and Methods}

This retrospective case-control study using metagenomics was conducted after obtaining approval from the Institutional Review Board of Seoul National University Hospital, Seoul, Korea (SNUH; No. 1612-102-816).

\subsection{Study Population}

Since June 2012, we have been collecting biological samples of patients scheduled to undergo surgery for adnexal masses for research purposes; under the patients' written informed consent, blood samples and cancer tissues are obtained the day before surgery and at the time of surgery, respectively, and then stored at the Human Biobank of SNUH.

For the present study, we identified relevant patients and obtained their frozen serum samples from the Human Biobank. Inclusion criteria for the study population were as follows: (1) older than 18 years; (2) underwent surgery for an adnexal mass between June 2012 and February 2018; and (3) pathologically diagnosed with either epithelial OC or benign ovarian tumor. Patients with the following conditions were excluded: (1) diagnosed with any malignancy other than OC synchronously or before the surgery; (2) neoadjuvant chemotherapy or targeted therapy before surgery; (3) borderline ovarian tumors; and (4) severe comorbidities, such as end-stage renal disease, uncontrolled diabetes mellitus, or long-term corticosteroid use.

In total, 166 patients with OC and 76 patients with benign ovarian tumors were included in this study. Through review of the medical records, we collected baseline characteristics including the age at diagnosis, BMI, comorbidities, and initial serum CA-125 levels. We also reviewed all patients' 
pathology results and collected information on the FIGO stage for the study group. Then, the patients' clinicopathologic characteristics were compared between the OC group and the benign ovarian tumor group. Metagenomic profiling was carried out with the patients' frozen serum samples according to the procedures described below.

\subsection{EV Isolation and DNA Extraction from Serum Samples}

We isolated EVs from the serum samples using the differential centrifugation method, as described previously [39]. In brief, serum samples were centrifuged at $3000 \mathrm{rpm}$ for $15 \mathrm{~min}$ at $4{ }^{\circ} \mathrm{C}$, and $100 \mathrm{uL}$ of the supernatant was mixed with $1 \times$ PBS, pH 7.4 (ML 008-01, Welgene, Republic of Korea). The floating particles were sunk through centrifugation at $10,000 \times \mathrm{g}$ for $1 \mathrm{~min}$ at $4{ }^{\circ} \mathrm{C}$. After centrifugation, bacteria and foreign particles were thoroughly eliminated by sterilizing the supernatant through a $0.22-u m$ filter.

To extract the DNA from the EVs' membranes, EVs separated from serum in the previous steps were boiled for $40 \mathrm{~min}$ at $100{ }^{\circ} \mathrm{C}$. To eliminate the remaining floating particles and debris, the supernatant was collected after $13,000 \mathrm{rpm}$ of centrifugation for $30 \mathrm{~min}$ at $4{ }^{\circ} \mathrm{C}$. EVs' DNA was extracted using a DNA isolation kit according to the standard protocol (PowerSoil DNA Isolation Kit, MO BIO, Carlsbad, CA, USA). The DNA from EVs in each sample was quantified by using the QIAxpert system (QIAGEN, Hilden, Germany).

\subsection{Microbial Metagenomic Analysis}

For $16 \mathrm{~S}$ rDNA gene-based metagenomic analysis, bacterial genomic DNA was amplified with 16S_V3_f (5'-TCGTCGGCAGCGTCAGATGTGTATAAGAGACAGCCTACGGGNGGCWGCAG-3') and 16S_V4_r(5'-GTCTCGTGGGCTCGGAGATGTGTATAAGAGACAGGACTACHVGGGTATCTAAT CC-3') primers, which are specific for the V3-V4 hypervariable regions of the 16S rDNA gene. The libraries were prepared using PCR products according to the MiSeq System guide (Illumina, San Diego, CA, USA) and quantified using a QIAxpert (QIAGEN, Hilden, Germany). Each amplicon was then quantified, and the equimolar ratio was set, pooled, and sequenced on a MiSeq (Illumina, San Diego, CA, USA) according to the manufacturer's recommendations.

\subsection{Analysis of Microbial Composition in the Microbiota}

Paired-end reads that matched the adapter sequences were trimmed by Cutadapt (version 1.1.6) [40]. The resulting FASTQ files containing paired-end reads were merged with CASPER and then quality filtered with Phred (Q) score-based criteria described by Bokulich [41,42]. Any reads shorter than $300 \mathrm{bp}$ after merging were also removed. To identify the chimeric sequences, a reference-based chimera detection step was conducted with VSEARCH against the Greengenes database [43]. Next, the sequence reads were clustered into operational taxonomic units (OTUs) using CD-HIT with a de novo clustering algorithm under a threshold of $97 \%$ sequence similarity. The representative sequences of the OTUs were finally classified using the Greengenes database (version 13.8) with UCLUST (parallel_assign_taxonomy_uclust.py script on QIIME (version 1.9.1) under default parameters) [44]. The Chao indices, an estimator of the richness of taxa per individual, were estimated to measure the diversity of each sample.

\subsection{Development of Diagnostic Models for Ovarian Cancer}

To construct and validate the diagnostic models for OC, we randomly divided the samples from each group into training and test sets in the ratio 2:1, considering the ratio of $O C$ and benign ovarian tumors in the total 242 samples. The values of each training and test set were transformed to a centered $\log$ ratio. Discovery of microbiome biomarkers and construction of diagnostic models were performed in the training set $(n=161)$, while validation of newly developed diagnostic models were performed in the test set $(n=81)$.

We filtered the genus if the zero proportion was more than $99 \%$. To identify specific microbiome biomarkers that were differentially distributed between the OC and benign ovarian tumor groups, 
we performed metagenomic analyses using eight statistical methods popularly used with the filtered count data: Wilcoxon, Metastats, EdgeR, DESeq2, ZIG, ZIBSeq, ANCOM, and CLR Perm. We used the abundance of the OTUs as the algorithms were developed based on the abundance data. Comparing the list of significant microbiome biomarkers identified by each statistical method, we chose biomarkers that overlapped as far as possible, because each method provides a different list of microbiome biomarkers, and most overlapped ones are expected to be highly plausible biomarkers.

We constructed several diagnostic models identifying OC from benign ovarian tumors by combining the microbiome biomarkers with patients' ages and serum CA-125 levels, and these models were validated in the test set. To evaluate the diagnostic performance of the developed models, each model's sensitivity, specificity, and AUC were calculated.

\subsection{Statistical Analysis}

Statistical analyses were performed to evaluate differences in the clinicopathologic characteristics between the two groups. The Student's $t$-test and Mann-Whitney U test were used to compare continuous variables, while the Pearson's chi-square test and Fisher's exact test were used to compare categorical variables. Shannon index was calculated to measure $\alpha$-diversities of the microbiota.

Summaries of the eight statistical methods that were applied to the metagenomic analyses are as follows: (1) The Wilcoxon rank sum test is the nonparametric type of the two-sample $t$-test, which uses the sum of ranks for observations. (2) Metastats compares the number of samples by group and the number of taxaons. Welch's $t$-test statistics were applied when the taxon count was larger than the number of samples. Otherwise, Fisher's exact test was used. (3) EdgeR and (4) DESeq2 methods are usually used in the analysis of RNA-sequencing data. As metagenome data is extracted from 16S rDNA, application of these methods has been often tried. Both methods are the negative binomial models; however, the difference between the two methods is that EdgeR uses a trimmed mean of M-values normalization, whereas DESeq2 uses a relative log expression normalization. (5) ZIG uses the log normal mixture model for the taxon count, taking sparsity on the OTU table into account. To overcome high false-positive rates, we adopted empirical Bayes shrinkage of parameter estimates. (6) ZIBSeq uses the beta mixture model for relative abundance. Relative abundance after total sum-scaling normalization was performed owing to the large number of zeros and results with the skewed distribution. (7) ANCOM was used to compare relative abundance of the OTUs; Wilcoxon rank sum test was used in comparisons of the two groups after the log-ratio transformation of all pairwise taxa. The Kruskal-Wallis test was used in comparisons of the three groups, and the Freidman test was used in comparisons of repetitive data. (8) CLR Perm fits the logistic model after the centered log-ratio transformation with count data to alleviate the sum to one constraint of relative abundance. The permutation test was adopted to decrease the false discovery rate.

R statistical software (version 3.4.4; R Foundation for Statistical Computing, Vienna, Austria; ISBN 3-900051-07-0; http://www.R-project.org) was used for the statistical analyses. A two-sided $p$-value below 0.05 was considered statistically significant.

\section{Conclusions}

In conclusion, we found that $16 \mathrm{~S}$ rDNA gene-based metagenomic analyses revealed differences in the metagenomic profiles of serum microbial EVs between patients with OC and those with benign ovarian tumors. We also developed a microbiome biomarker-based diagnostic model differentiating OC from benign ovarian tumors and found that the serum microbiome may play a role in the early detection of OC. Further prospective studies are warranted to validate these results.

Supplementary Materials: The following are available online at http://www.mdpi.com/2072-6694/12/5/1309/s1: Figure S1. Flow diagram illustrating the overall study design. Figure S2. Comparisons of genus-level $\alpha$-diversity between the two groups. Figure S3. Genus-level multidimensional plots. 
Author Contributions: Conceptualization, T.P. and Y.S.S.; Data curation, S.I.K., J.Y., H.J., M.L., H.S.K. and Y.-K.K.; Formal analysis, S.I.K., N.K., S.L., J.Y., Y.-K.K., T.P. and Y.S.S.; Funding acquisition, Y.S.S.; Investigation, S.I.K., N.K., S.L., J.Y., H.J., M.L., H.S.K., D.N.D., Y.-K.K. and Y.S.S.; Methodology, N.K., S.L., J.Y. and T.P.; Project administration, Y.S.S.; Resources, Y.S.S.; Software, N.K., J.Y. and T.P.; Supervision, T.P. and Y.S.S.; Validation, S.I.K., HA.J., M.L., H.S.K., D.N.D. and T.P.; Visualization, N.K.; Writing-original draft, S.I.K., N.K., J.Y. and T.P.; Writing一review \& editing, S.K., N.K., S.L., J.Y., H.J., M.L., H.S.K., D.N.D., Y.-K.K., T.P. and Y.S.S. All authors have read and agreed to the published version of the manuscript.

Funding: This work was supported by a grant from the Korea Health Technology R\&D Project through the Korea Health Industry Development Institute (KHIDI), funded by the Ministry of Health and Welfare, Republic of Korea (No. HI16C2037).

Acknowledgments: The biospecimens for this study were provided by the Seoul National University Hospital Human Biobank, a member of the National Biobank of Korea, which is supported by the Ministry of Health and Welfare. All samples, derived from the National Biobank of Korea, were obtained with informed consent under institutional review board-approved protocols.

Conflicts of Interest: The authors declare no conflicts of interest.

\section{References}

1. Bray, F.; Ferlay, J.; Soerjomataram, I.; Siegel, R.L.; Torre, L.A.; Jemal, A. Global cancer statistics 2018: GLOBOCAN estimates of incidence and mortality worldwide for 36 cancers in 185 countries. CA A Cancer J. Clin. 2018, 68, 394-424. [CrossRef]

2. Siegel, R.L.; Miller, K.D.; Jemal, A. Cancer statistics, 2019. CA Cancer J. Clin. 2019, 69, 7-34. [CrossRef] [PubMed]

3. Lim, M.C.; Won, Y.-J.; Ko, M.J.; Kim, M.; Shim, S.-H.; Suh, D.H.; Kim, J.-W. Incidence of cervical, endometrial, and ovarian cancer in Korea during 1999-2015. J. Gynecol. Oncol. 2019, 30. [CrossRef] [PubMed]

4. Baldwin, L.A.; Huang, B.; Miller, R.W.; Tucker, T.; Goodrich, S.T.; Podzielinski, I.; DeSimone, C.P.; Ueland, F.R.; Van Nagell, J.R.; Seamon, L.G. Ten-Year Relative Survival for Epithelial Ovarian Cancer. Obstet. Gynecol. 2012, 120, 612-618. [CrossRef] [PubMed]

5. Myers, E.R.; Bastian, L.A.; Havrilesky, L.J.; Kulasingam, S.L.; Terplan, M.S.; Cline, K.E.; Gray, R.N.; McCrory, D.C. Management of adnexal mass. Evid. Rep. Assess. 2006, 2006, 1-145.

6. Jacobs, I.; Oram, D.; Fairbanks, J.; Turner, J.; Frost, C.; Grudzinskas, J.G. A risk of malignancy index incorporating CA 125, ultrasound and menopausal status for the accurate preoperative diagnosis of ovarian cancer. BJOG: Int. J. Obstet. Gynaecol. 1990, 97, 922-929. [CrossRef]

7. Moore, R.G.; McMeekin, D.S.; Brown, A.K.; DiSilvestro, P.; Miller, M.C.; Allard, W.J.; Gajewski, W.; Kurman, R.; Bast, R.C.; Skates, S.J. A novel multiple marker bioassay utilizing HE4 and CA125 for the prediction of ovarian cancer in patients with a pelvic mass. Gynecol. Oncol. 2008, 112, 40-46. [CrossRef]

8. Karlsen, M.A.; Sandhu, N.; Høgdall, C.; Christensen, I.J.; Nedergaard, L.; Lundvall, L.; Engelholm, S.A.; Pedersen, A.T.; Hartwell, R.; Lydolph, M.; et al. Evaluation of HE4, CA125, risk of ovarian malignancy algorithm (ROMA) and risk of malignancy index (RMI) as diagnostic tools of epithelial ovarian cancer in patients with a pelvic mass. Gynecol. Oncol. 2012, 127, 379-383. [CrossRef]

9. Liest, A.-L.; Omran, A.S.; Mikiver, R.; Rosenberg, P.; Uppugunduri, S. RMI and ROMA are equally effective in discriminating between benign and malignant gynecological tumors: A prospective population-based study. Acta Obstet. Gynecol. Scand. 2018, 98, 24-33. [CrossRef]

10. Van Gorp, T.; Cadron, I.; Despierre, E.; Daemen, A.; Leunen, K.; Amant, F.; Timmerman, D.; De Moor, B.; Vergote, I. HE4 and CA125 as a diagnostic test in ovarian cancer: Prospective validation of the Risk of Ovarian Malignancy Algorithm. Br. J. Cancer 2011, 104, 863-870. [CrossRef]

11. Lycke, M.; Kristjansdottir, B.; Sundfelt, K. A multicenter clinical trial validating the performance of HE4, CA125, risk of ovarian malignancy algorithm and risk of malignancy index. Gynecol. Oncol. 2018, 151, 159-165. [CrossRef] [PubMed]

12. Dayyani, F.; Uhlig, S.; Colson, B.; Simon, K.; Rolny, V.; Morgenstern, D.; Schlumbrecht, M. Diagnostic Performance of Risk of Ovarian Malignancy Algorithm Against CA125 and HE4 in Connection With Ovarian Cancer: A Meta-analysis. Int. J. Gynecol. Cancer 2016, 26, 1586-1593. [CrossRef] [PubMed]

13. Geng, J.; Fan, H.; Tang, X.; Zhai, H.; Zhang, Z. Diversified pattern of the human colorectal cancer microbiome. Gut Pathogens 2013, 5, 2. [CrossRef] [PubMed]

14. Schwabe, R.F.; Jobin, C. The microbiome and cancer. Nat. Rev. Cancer 2013, 13, 800-812. [CrossRef]

15. Bultman, S.J. Emerging roles of the microbiome in cancer. Carcinog 2013, 35, 249-255. [CrossRef] 
16. Ahn, J.; Sinha, R.; Pei, Z.; Dominianni, C.; Wu, J.; Shi, J.; Goedert, J.J.; Hayes, R.B.; Yang, L. Human gut microbiome and risk for colorectal cancer. J. Natl. Cancer Inst. 2013, 105, 1907-1911. [CrossRef]

17. Bhatt, A.P.; Redinbo, M.R.; Bultman, S.J. The role of the microbiome in cancer development and therapy. CA Cancer J. Clin. 2017, 67, 326-344. [CrossRef]

18. Raza, M.H.; Gul, K.; Arshad, A.; Riaz, N.; Waheed, U.; Rauf, A.; Aldakheel, F.; Alduraywish, S.; Rehman, M.U.; Abdullah, M.; et al. Microbiota in cancer development and treatment. J. Cancer Res. Clin. Oncol. 2018, 145, 49-63. [CrossRef]

19. Kuehn, M. Bacterial outer membrane vesicles and the host-pathogen interaction. Genome Res. 2005, 19, 2645-2655. [CrossRef]

20. Roier, S.; Zingl, F.G.; Cakar, F.; Schild, S. Bacterial outer membrane vesicle biogenesis: A new mechanism and its implications. Microb. Cell 2016, 3, 257-259. [CrossRef]

21. Zhou, B.; Sun, C.; Huang, J.; Xia, M.; Guo, E.; Li, N.; Lu, H.; Shan, W.; Wu, Y.; Li, Y.; et al. The biodiversity Composition of Microbiome in Ovarian Carcinoma Patients. Sci. Rep. 2019, 9, 1691. [CrossRef] [PubMed]

22. Pepper, I.L.; Gerba, C.P.; Gentry, T.J. Environmental Microbiology, 3rd ed.; Academic press: San Diego, CA, USA, 2014.

23. Kinross, J.M.; Darzi, A.W.; Nicholson, J. Gut microbiome-host interactions in health and disease. Genome Med. 2011, 3, 14. [CrossRef] [PubMed]

24. Clemente, J.C.; Ursell, L.K.; Parfrey, L.W.; Knight, R. The impact of the gut microbiota on human health: An integrative view. Cell 2012, 148, 1258-1270. [CrossRef] [PubMed]

25. Walther-Antonio, M.; Chen, J.; Multinu, F.; Hokenstad, A.; Distad, T.J.; Cheek, E.H.; Keeney, G.L.; Creedon, D.J.; Nelson, H.; Mariani, A.; et al. Potential contribution of the uterine microbiome in the development of endometrial cancer. Genome Med. 2016, 8, 122. [CrossRef] [PubMed]

26. Peleg, A.; Seifert, H.; Paterson, D.L. Acinetobacter baumannii: Emergence of a Successful Pathogen. Clin. Microbiol. Rev. 2008, 21, 538-582. [CrossRef] [PubMed]

27. Prabhash, K.; Medhekar, A.; Ghadyalpatil, N.; Noronha, V.; Biswas, S.; Kurkure, P.; Nair, R.; Kelkar, R. Blood stream infections in cancer patients: A single center experience of isolates and sensitivity pattern. Indian J. Cancer 2010, 47, 184. [CrossRef]

28. Chiang, M.-C.; Kuo, S.-C.; Chen, S.-J.; Yang, S.-P.; Lee, Y.-T.; Chen, T.-L.; Fung, C.-P. Clinical characteristics and outcomes of bacteremia due to different genomic species of Acinetobacter baumannii complex in patients with solid tumors. Infection 2011, 40, 19-26. [CrossRef]

29. Takeuchi, O.; Akira, S. Toll-like receptors; their physiological role and signal transduction system. Int. Immunopharmacol. 2001, 1, 625-635. [CrossRef]

30. Knapp, S.; Wieland, C.W.; Florquin, S.; Pantophlet, R.; Dijkshoorn, L.; Tshimbalanga, N.; Akira, S.; Van Der Poll, T. Differential Roles of CD14 and Toll-like Receptors 4and 2 in MurineAcinetobacterPneumonia. Am. J. Respir. Crit. Care Med. 2006, 173, 122-129. [CrossRef]

31. Erridge, C.; Moncayo-Nieto, O.L.; Morgan, R.; Young, M.; Poxton, I.R. Acinetobacter baumannii lipopolysaccharides are potent stimulators of human monocyte activation via Toll-like receptor 4 signalling. J. Med. Microbiol. 2007, 56, 165-171. [CrossRef]

32. Kim, C.; Jeong, Y.-J.; Lee, J.; Jeon, S.-J.; Park, S.-R.; Kang, M.-J.; Park, J.-H.; Park, J.-H. Essential role of toll-like receptor 4 in Acinetobacter baumannii-induced immune responses in immune cells. Microb. Pathog. 2013, 54, 20-25. [CrossRef] [PubMed]

33. Jun, S.H.; Lee, J.H.; Kim, B.R.; Kim, S.I.; Park, T.I.; Lee, J.C.; Lee, Y.-C. Acinetobacter baumannii Outer Membrane Vesicles Elicit a Potent Innate Immune Response via Membrane Proteins. PLoS ONE 2013, 8, e71751. [CrossRef] [PubMed]

34. Nho, J.S.; Jun, S.H.; Oh, M.H.; Park, T.I.; Choi, C.W.; Kim, S.I.; Choi, C.H.; Lee, J.C. Acinetobacter nosocomialis secretes outer membrane vesicles that induce epithelial cell death and host inflammatory responses. Microb. Pathog. 2015, 81, 39-45. [CrossRef] [PubMed]

35. Zhou, M.; McFarland-Mancini, M.M.; Funk, H.M.; Husseinzadeh, N.; Mounajjed, T.; Drew, A. Toll-like receptor expression in normal ovary and ovarian tumors. Cancer Immunol. Immunother. 2009, 58, 1375-1385. [CrossRef]

36. Kelly, M.G.; Alvero, A.; Chen, R.; Silasi, D.-A.; Abrahams, V.M.; Chan, S.; Visintin, I.; Rutherford, T.; Mor, G. TLR-4 Signaling Promotes Tumor Growth and Paclitaxel Chemoresistance in Ovarian Cancer. Cancer Res. 2006, 66, 3859-3868. [CrossRef] 
37. Sugiyama, T.; Okamoto, A.; Enomoto, T.; Hamano, T.; Aotani, E.; Terao, Y.; Suzuki, N.; Mikami, M.; Yaegashi, N.; Kato, K.; et al. Randomized Phase III Trial of Irinotecan Plus Cisplatin Compared With Paclitaxel Plus Carboplatin As First-Line Chemotherapy for Ovarian Clear Cell Carcinoma: JGOG3017/GCIG Trial. J. Clin. Oncol. 2016, 34, 2881-2887. [CrossRef]

38. Kim, S.I.; Lim, M.C.; Lim, J.; Won, Y.-J.; Seo, S.-S.; Kang, S.; Park, S.-Y. Incidence of epithelial ovarian cancer according to histologic subtypes in Korea, 1999 to 2012. J. Gynecol. Oncol. 2016, 27. [CrossRef]

39. Rhee, S.J.; Kim, H.; Lee, Y.; Lee, H.J.; Park, C.H.K.; Yang, J.; Kim, Y.-K.; Kym, S.; Ahn, Y.M. Comparison of serum microbiome composition in bipolar and major depressive disorders. J. Psychiatr. Res. 2020, 123, 31-38. [CrossRef]

40. Martin, M. Cutadapt removes adapter sequences from high-throughput sequencing reads. EMBnet J. 2011, 17, 10. [CrossRef]

41. Bokulich, N.A.; Subramanian, S.; Faith, J.J.; Gevers, D.; Gordon, J.I.; Knight, R.; Mills, D.A.; Caporaso, J.G. Quality-filtering vastly improves diversity estimates from Illumina amplicon sequencing. Nat. Methods 2013, 10, 57-59. [CrossRef]

42. Kwon, S.; Lee, B.; Yoon, S. CASPER: Context-aware scheme for paired-end reads from high-throughput amplicon sequencing. BMC Bioinform. 2014, 15, S10. [CrossRef] [PubMed]

43. Rognes, T.; Flouri, T.; Nichols, B.; Quince, C.; Mahé, F. VSEARCH: A versatile open source tool for metagenomics. Peer] 2016, 4, e2584. [CrossRef] [PubMed]

44. Caporaso, J.G.; Kuczynski, J.; Stombaugh, J.; Bittinger, K.; Bushman, F.D.; Costello, E.K.; Fierer, N.; Peña, A.G.; Goodrich, J.K.; Gordon, J.I.; et al. QIIME allows analysis of high-throughput community sequencing data. Nat. Methods 2010, 7, 335-336. [CrossRef] [PubMed]

(C) 2020 by the authors. Licensee MDPI, Basel, Switzerland. This article is an open access article distributed under the terms and conditions of the Creative Commons Attribution (CC BY) license (http://creativecommons.org/licenses/by/4.0/). 